\title{
Down-regulation of long non-coding RNA XIST aggravates sepsis-induced lung injury by regulating miR-16-5p
}

\author{
Xiaofei Song ${ }^{1} \cdot$ Linyu $\mathrm{Li}^{2} \cdot$ Yaying Zhao ${ }^{3} \cdot$ Yucheng Song $^{1}$ (i) \\ Received: 19 August 2020 / Accepted: 27 April 2021 / Published online: 12 May 2021 \\ (c) Japan Human Cell Society 2021
}

\begin{abstract}
This study aims to explain the role and related mechanisms of long non-coding RNA (lncRNA) X inactive specific transcript (XIST) in sepsis-induced acute lung injury (ALI). The in vivo septic models and in vitro septic model were established. In animal models, the lung injury of the rats was evaluated after XIST was overexpressed. In cell models, the effects of XIST and microRNA (miR)-16-5p on ALI was detected by MTT assay, Western blot and ELISA. The interaction between XIST and miR-16-5p was investigated by bioinformatics analysis, dual-luciferase reporter assay, RIP assay and RNA pull-down assay. We found that XIST expression was down-regulated in lung tissues of septic rats and lipopolysaccharide-stimulated cells, while the expression of miR-16-5p was up-regulated. Down-regulation of XIST significantly promoted pulmonary edema, increased the levels of TNF- $\alpha$, IL- $1 \beta$ and malondialdehyde, inhibited the cell viability and decreased the level of superoxide dismutase. Mechanistically, it was confirmed that XIST could sponge miR-16-5p, and thus repress its expression, and the transfection of miR-16-5p mimics could reverse the effects of XIST over-expression in the cell model. Collectively, it is concluded that XIST reduces sepsis-induced ALI via regulating miR-16-5p.
\end{abstract}

Keywords XIST $\cdot$ miR-16-5p $\cdot$ Sepsis $\cdot$ Lung injury

Yucheng Song

zhen25525525585@163.com

Xiaofei Song

sigunying84711@163.com

Linyu Li

hanxing2588588@163.com

Yaying Zhao

fu258558151148@163.com

1 Department of Gastrointestinal Surgery, Henan Provincial People's Hospital, Zhengzhou University People's Hospital, Medical College of Henan University, Weiwu Road No. 7, Zhengzhou 450003, Henan Province, China

2 Department of Scientific Research, Sanquan College of Xinxiang Medical University, Xinxiang 453003, China

3 Department of Disinfection Supply Center, Henan Provincial People's Hospital, Zhengzhou University People's Hospital, Medical College of Henan University, Zhengzhou 450003, China

\section{Introduction}

Sepsis is the systemic response to infection and is defined as the presence of systemic inflammatory response syndrome (SIRS), characterized by a cytokine-mediated hyper-inflammatory phase and a subsequent immune-suppressive phase [1]. As one of the most serious complications of sepsis, acute lung injury (ALI) is prone to develop into acute respiratory distress syndrome (ARDS). The mortality of ALI during sepsis is as high as $25.6 \%$ [2]. Clarifying the mechanism of sepsis-mediated ALI is of great significance for early diagnosis, therapeutic effect evaluation and prognosis prediction of the disease.

Long non-coding RNA (lncRNA) is a class of transcripts with a length of over $200 \mathrm{nt}$, which cannot be translated into proteins [3]. Recent studies reported that in the process of systemic inflammatory response, lncRNA plays a pivotal role through regulating multiple pathways, modulating the proliferation and differentiation of inflammatory cells, changing the expression of inflammation-related genes, and mediating the production and release of cytokines [4, 5]. In addition, accumulating evidence has demonstrated that IncRNAs take part in the progression of sepsis [6, 7]. X 
inactive specific transcript (XIST) has been previously found to be up-regulated in several cancers and can facilitate proliferation and metastasis of cancer cells [8,9]. Additionally, XIST expression is increased in the bronchoalveolar lavage fluid of primary graft dysfunction (PGD) patients after lung transplantation, and overexpression of XIST aggravates PGD after lung transplantation [10]. However, the function and mechanism of XIST in sepsis-induced ALI still remain unclear.

MicroRNAs (miRNAs) are non-coding RNAs (ncRNAs) with highly conserved sequences. They can degrade mRNA or inhibit the translation through specific binding with the 3' untranslated region (3'UTR) [11, 12]. Moreover, miRNAs regulate a series of biological processes, including cell proliferation, differentiation, apoptotic signal transduction and so on [11-13]. Importantly, a number of studies demonstrate that miRNAs are involved in the pathogenesis of sepsis $[14,15]$. Recent studies reveal that miR-16-5p regulates the progression of cancers $[16,17]$, while the function of miR$16-5 \mathrm{p}$ in sepsis-induced ALI is still obscure.

In the present study, with in vitro and in vivo experiments, we demonstrated that the expression of XIST was significantly down-regulated in the lung tissues of septic rats and cell model of sepsis. Functionally and mechanistically, it was demonstrated that XIST could regulate the apoptosis of pulmonary epithelial cells by regulating miR-16-5p. This work provides a new theoretical basis for clinical treatment of sepsis-induced ALI.

\section{Methods and materials}

\section{Establishment of a septic rat model}

All of the procedures in this study were approved by the Animal Studies Committee of Henan Provincial People's Hospital (Approval No. 2014-A11). The rat model with sepsis was established by cecum ligation and puncture (CLP) as previously described [18]. 21 male Sprague-Dawley (SD) rats (age 7-8 weeks; weight 200-250 g) were randomly divided into sham group, CLP group and CLP + recombinant adeno-associated virus carrying XIST overexpression vector (CLP + RAAV-XIST) group ( $n=7$ rats in each group). The XIST overexpressing recombinant adeno-associated virus was purchased from Vigene Bioscience (Jinan, China), and RAAV-GFP served as the negative control, which was packed with RAAV virus and empty vector. Full length of XIST was cloned into the AAV vector pAAV-CMV-ireshrEGFP to construct the AAV transgene plasmid pAAVCMV-XIST-ires-hrEGFP. pAAV-RC9, pHelper, and the AAV transgene plasmid were co-transfected into HEK-293T cells. At $72 \mathrm{~h}$ after transfection, the HEK-293T cells were incubated with Benzonase endonuclease (Sigma-Aldrich,
St. Louis, MO, USA) at $37{ }^{\circ} \mathrm{C}$ for $1 \mathrm{~h}$, and then the RAAV particles were purified by a heparin column (Sigma-Aldrich, St. Louis, MO, USA). After the purification, RAAV-XIST and RAAV-GFP were injected into CLP rats via the tail vein.

\section{Cell culture and transfection}

Human small airway epithelial cells (HSAECs) and HEK293T cells were purchased from the Cell Bank of Type Culture Collection of the Chinese Academy of Sciences (Shanghai, China). HSAECs were cultured in Dulbecco's Modified Eagle's Medium (DMEM; Invitrogen, Carlsbad, CA, USA) supplemented with $10 \%$ fetal bovine serum (FBS; Gibco, Carlsbad, CA, USA) at $37{ }^{\circ} \mathrm{C}$ in $5 \% \mathrm{CO}_{2}$ and saturated humidity. In lipopolysaccharide (LPS) group, HSAECs were treated with $10 \mu \mathrm{g} / \mathrm{ml}$ LPS (Sigma-Aldrich, St. Louis, MO, USA) for $24 \mathrm{~h}$ to establish the sepsis-induced ALI model in vitro. MiR-16-5p mimics and its control (miR-16-5p NC), miR-16-5p inhibitors, XIST overexpression plasmids (XIST), XIST short hairpin RNA (shRNA) (sh-XIST-1: 5'-GCTGACTACCTGAGATTTAAG-3'; sh-XIST-2: 5'-GCTCTTGAACAGTTAATTTGC-3'), and negative controls (sh-NC: GGGACUCUCGGUAUGUAAGAUU) were purchased from RiboBio Co., Ltd. (Guangzhou, China). The transfection was conducted by Lipofectamine ${ }^{\mathrm{TM}} 3000$ transfection reagent (Invitrogen, Carlsbad, CA, USA) in accordance with the manufacturer's instructions.

\section{Lung wet-to-dry (W/D) weight ratio}

The lung W/D weight ratio was used to evaluate the pulmonary edema of the rats. After the rats were killed, the fresh right upper lung tissue was obtained and weighed for wet weight (W) and then dried for at least $24 \mathrm{~h}$ in an oven at $180^{\circ} \mathrm{C}$ to measure dry weight (D), and then W/D weight ratio was calculated.

\section{Enzyme-linked immunosorbent assay (ELISA)}

The lung tissues samples of rats in different groups were homogenized and centrifuged $(14,000 \times g, 5 \mathrm{~min})$ to collect the supernatant. Levels of tumor necrosis factor- $\alpha$ (TNF- $\alpha)$ and interleukin-1 $\beta$ (IL-1 $\beta$ ) in cell culture supernatant and lung tissues were detected by the corresponding ELISA kit (Lianke Biotechnology Co., Ltd., Hangzhou, China) according to manufacturer's instructions.

\section{Detection of superoxide dismutase (SOD) and malondialdehyde (MDA)}

MDA content was determined by the thiobarbituric acid method, whereas the SOD activity was evaluated by the xanthine oxidase method. The MDA content and SOD activity 
in lung tissues and cell supernatant were measured with a spectrometer using commercially available kits (JianCheng Bioengineering Institute, Nanjing, China).

\section{Terminal deoxynucleotidyl transferase (TdT)-mediated dUTP nick-end labeling (TUNEL) assay}

Lung tissue sections were fixed with $4 \%$ paraformaldehyde for $30 \mathrm{~min}$ and incubated with $0.5 \%$ Triton X-100 for $10 \mathrm{~min}$. Next, the samples were incubated at room temperature for 60 min with $50 \mu \mathrm{L}$ of TUNEL Apoptosis Detection kit (Beyotime, Hangzhou, China) according to the manufacturer's protocols and then washed with PBS three times. The nuclei were stained with 4',6-diamidino-2-phenylindole (DAPI) for $30 \mathrm{~min}$. Next the images of the cells were captured by a laser confocal microscopy (Carl Zeiss AG, Oberkochen, Germany).

\section{Quantitative reverse transcription polymerase chain reaction ( $R T-q P C R)$}

TRIzol reagent (Invitrogen, Carlsbad, CA, USA) was used to extract the total RNA from the lung tissues and HSAECs, with the purity and concentration of total RNA detected by an ultraviolet (UV) spectrophotometer. Then the total RNA was reverse-transcribed into cDNA with SuperScript First-Strand cDNA System (Invitrogen, Grand Island, NY, USA). With cDNA as the template, SYBR premix EX TAQ II (Takara, Dalian, China) was used to perform RT-qPCR on the Applied Biosystems 7500 Real-Time PCR System (Applied Biosystems, Foster City, CA, USA). GAPDH was used as the standard internal reference of XIST, and U6 as that of miR-16-5p. The relative gene expressions were calculated by $2^{-\Delta \Delta \mathrm{CT}}$ method. The specific primer sequences are listed in Table 1. To identify the subcellular location of XIST, the RNA were separately extracted from the cytosol and nucleus of HSAECs with NE-PER ${ }^{\mathrm{TM}}$ Nuclear and Cytoplasmic Extraction Reagents (Thermo Fisher Scientific, Waltham, MA, USA) and the RNeasy Kit (Qiagen,

Table 1 Primer sequences used of RT-qPCR

\begin{tabular}{|c|c|}
\hline Name & Primer sequences \\
\hline \multirow[t]{2}{*}{ XIST } & Forward: 5'-ACGCTGCATGTGTCCTTAG-3' \\
\hline & Reverse: 5'-GAGCCTCTTATAGCTGTTTG-3' \\
\hline \multirow[t]{2}{*}{ GAPDH } & Forward: 5'-GCACCGTCAAGGCTGAGAAC-3' \\
\hline & Reverse: 5'-ATGGTGGTGAAGACGCCAGT-3' \\
\hline \multirow[t]{2}{*}{$\operatorname{miR}-16-5 p$} & Forward: 5'-TAGCAGCACGTAAATATTGGCG-3' \\
\hline & Reverse: 5'-TGCGTGTCGTGGAGTC-3' \\
\hline \multirow[t]{2}{*}{ U6 } & Forward: 5'-CTCGCTTCGGCAGCACA-3' \\
\hline & Reverse: 5'-AACGCTTCACGAATTTGCGT-3' \\
\hline
\end{tabular}

Shanghai, China). Next, XIST expression in the two subcellular fractions was detected by RT-qPCR.

\section{MTT assay}

HSAECs in different groups were inoculated into 96-well plates at a density of $1 \times 10^{3}$ cells/well. After cultured for $24 \mathrm{~h}$, the cells were treated with LPS $(10 \mu \mathrm{g} / \mathrm{mL})$ for $24 \mathrm{~h}$, and $20 \mu \mathrm{L}$ of MTT solution was added, and the cells were incubated for $4 \mathrm{~h}$. Following that, dimethyl sulfoxide (DMSO) was added into the wells, and the formazan was dissolved. Next, the absorbance of each well was detected with a microplate reader, to examine the viability of the cells.

\section{Western blot}

HSAECs in each group were lysed on ice with RIPA lysis buffer (Beyotime, Shanghai, China) for $30 \mathrm{~min}$, and then centrifuged at $14,000 \mathrm{rpm}$ for $15 \mathrm{~min}$ at $4{ }^{\circ} \mathrm{C}$. The BCA Protein Assay kit (Thermo Fisher Scientific, Waltham, MA, USA) was utilized to detect the protein concentration. Subsequently, sodium dodecyl sulfate-polyacrylamide gel electrophoresis (SDS-PAGE) was performed, and the protein was transferred to polyvinylidene difluoride (PVDF) membranes, which were then blocked in 5\% skimmed milk at room temperature for $2 \mathrm{~h}$. After that, anti-Bcl-2 antibody (1:1000; ab196495; Abcam, Cambridge, UK), and anti-Bax antibody (1:1000; ab53154; Abcam, Cambridge, UK) were added, respectively, and then the membranes were incubated at $4{ }^{\circ} \mathrm{C}$ for $12 \mathrm{~h}$. After the membranes were rinsed with TBS, the membranes were incubated at room temperature for $1 \mathrm{~h}$ with the secondary antibody (Beyotime, Shanghai, China). At last, the protein bands were developed by the enhanced chemiluminiscence (ECL) kit (Beyotime, Shanghai, China).

\section{Dual luciferase reporter gene assay}

Wild-type (WT) XIST sequence was amplified and inserted into pmirGLO dual-luciferase miRNA target expression vectors (Promega Corp., Madison, WI, USA) to construct the reporter vector pmirGLO-XIST-WT (XIST WT). GeneArt ${ }^{\mathrm{TM}}$ Site-Directed Mutagenesis PLUS System (Thermo Fisher Scientific, Inc., MA, USA) was used to mutate the presumptive binding sites of miR-16-5p in XIST. Next, the mutant (MUT) XIST sequence was inserted into the pmirGLO vector to construct reporter vector pmirGLO-XIST-MUT (XIST MUT1, XIST MUT2, and XIST MUT1\&2). Next, the reporter vectors and miR-16-5p or miR-NC were cotransfected into HKE-293T cells and the cells were cultured for $48 \mathrm{~h}$. Following that, the luciferase activity was monitored by a Dual-Luciferase Reporter Assay System (Promega, Madison, WI, USA). 


\section{RNA immunoprecipitation (RIP) assay}

The Magna RIP RNA-Binding Protein Immunoprecipitation Kit (Millipore, Billerica, MA, USA) was used to perform RIP assay. Briefly, the HSAECs were lysed in lysis buffer. Next, the mixtures were incubated with magnetic beads coupled with anti-Argonaute 2 (Ago2) or IgG (Millipore, Billerica, MA, USA) antibody for $12 \mathrm{~h}$ at room temperature. After the co-precipitated RNA was isolated from the immunoprecipitate, the expression of XIST and miR-16-5p were detected by RT-qPCR.

\section{RNA pull-down}

Biotin-labeled XIST was generated with biotin RNA-labeled mixtures (Roche, Basel, Switzerland) and T7-RNA polymerase (Roche, Basel, Switzerland). Then HSAECs were lysed in RIP buffer, and then mixed with biotin-marked XIST, and the mixture was incubated at $4{ }^{\circ} \mathrm{C}$ for $1 \mathrm{~h}$. Next, magnetic beads were added and incubated at room temperature for $1 \mathrm{~h}$. After the complex was isolated, Ago2 was detected by Western blot, and the expression of miR-16-5p was measured by RT-qPCR.

\section{Hematoxylin-eosin (HE) staining}

The paraffin-coated lung tissues of rats were sliced, dewaxed and rehydrated. Next, the sections were stained with hematoxylin solution for $5 \mathrm{~min}$, and then incubated in 5\% acetic acid, and then rinsed with tap water. Next, the sections were stained with eosin solution for $3 \mathrm{~min}$ and followed by dehydration with graded alcohol and clearing in xylene. At last, the sections were sealed, and the morphology of lung tissue was observed under a microscope. Lung injury scores were assessed by two independent pathologists as follows: 0 indicating no injury, 1 indicating 25\% lung injury, 2 indicating 50\% injury, 3 indicating $75 \%$ injury, and 4 indicating total lung injury.

\section{Statistical analysis}

In the present study, SPSS 20.0 statistical software was used to analyze the experimental data, which were expressed as mean \pm standard deviation $(x \pm s)$. Student's $t$ test was employed for making comparisons between the two samples, and one-way analysis of variance (ANOVA) was used for comparing multiple samples. $P<0.05$ was considered statistically significant.

\section{Results}

\section{XIST is down-regulated in CLP mice and can attenuates lung injury}

First of all, we detected the difference in miRNA expression profile in lung tissues between mice treated with LPS and the mice without LPS treatment, by analyzing GSE18341 with GEO2R tool. The results showed that XIST was significantly down-modulated in the lung tissues of the mice treated with LPS, suggesting XIST could probably participate in the lung injury induced by LPS (Fig. 1a, b). Then, we established sepsis-induced ALI models with CLP method, and it was found that compared with the sham group, XIST was down-regulated in the lung tissues of CLP group; compared with the CLP group, XIST was up-regulated in CLP + RAAV-XIST group (Fig. 1c). The lung W/D weight ratio of the rats notably increased in CLP group, suggesting that pulmonary edema was aggravated during sepsis, while the restoration of XIST decreased the lung W/D weight ratio (Fig. 1d). Additionally, compared with the sham group, TNF-a, IL- $1 \beta$ and MDA levels were up-regulated in the lung tissues of the rats in CLP group, SOD level was down-regulated, while XIST restoration reversed this effect (Fig. 1e, f). TUNEL assay suggested that the apoptosis in the lung tissues of CLP group was significantly severer than that of the sham group, while it was ameliorated by XIST restoration (Fig. 1g). In CLP group, HE staining showed that the diffuse hyaline membrane formation in the alveolar spaces and the alveolar structure of the rats was damaged, which suggested severe injury and inflammation; in CLP + RAAV-XIST group, the lung injury were obviously ameliorated (Fig. 1h). Additionally, XIST was mainly enriched in the cytoplasm, suggesting it could probably exert its biological effects via functioning as a molecular sponge for miRNAs (Fig. 1i).

\section{Effects of XIST on the viability and apoptosis of HSAECs treated with LPS}

To further determine the role of XIST in septic ALI, HSAECs were treated with LPS to establish an in vitro model. RT-qPCR showed that the expression of XIST in LPS-induced HSAECs was significantly down-regulated, and remarkably decreased in a time-dependent manner (Fig. 2a). Moreover, we transfected XIST overexpression, sh-XIST-1 and sh-XIST-2 into HSAMCs, respectively, before LPS treatment (Fig. 2b). MTT assay, Western blot and ELISA showed that, after the overexpression of XIST, the viability of HSAECs was notably increased, the Bax, TNF- $\alpha$, IL- $1 \beta$ and MDA levels were markedly decreased, 

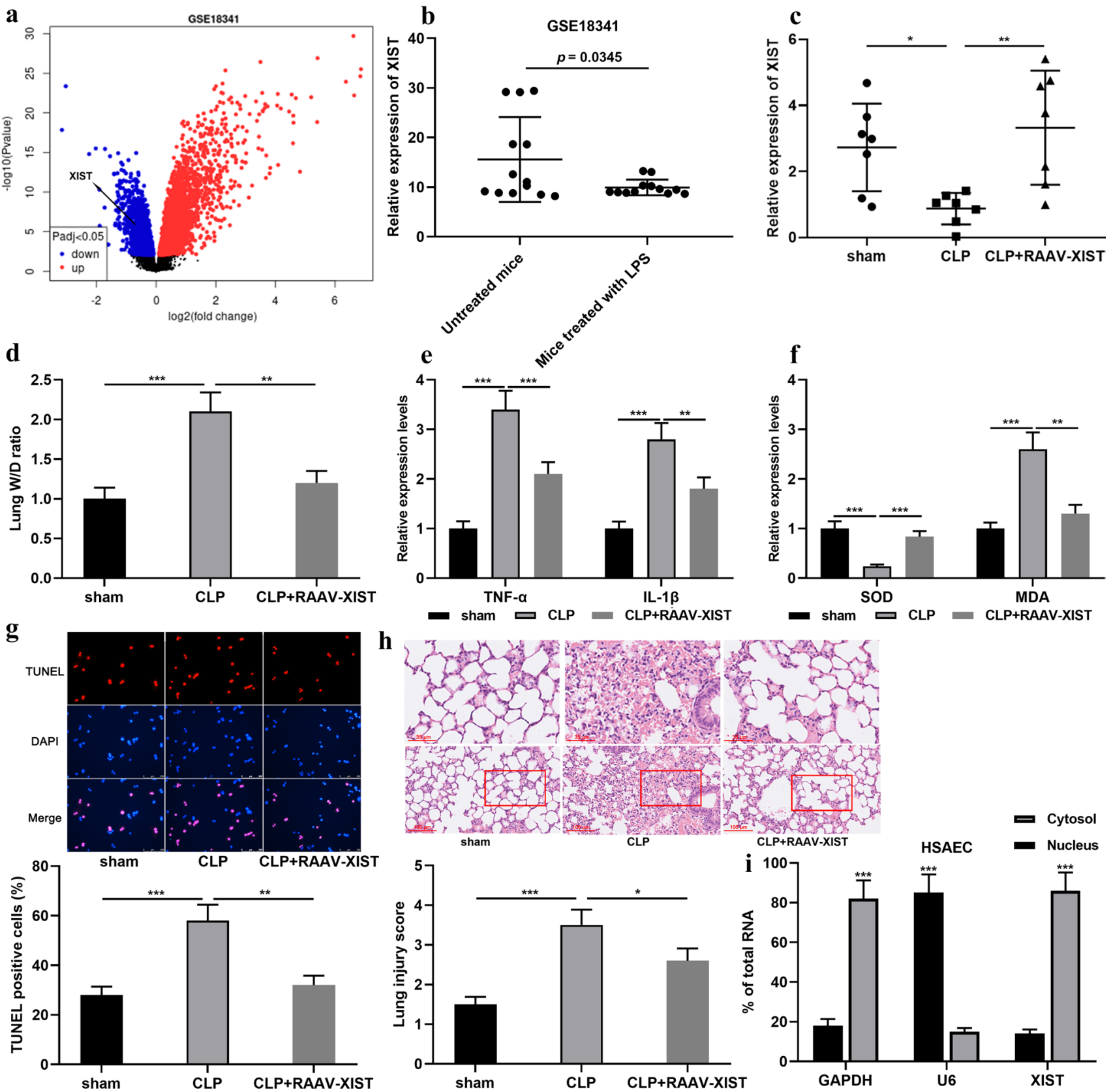

Fig. 1 XIST was down-regulated in the lung tissues of septic rats and it ameliorated lung injury induced by sepsis. a, b The lncRNA expression dataset GSE18341 was downloaded from GEO database. Volcano plot was plotted for the visualization of the differentially expressed lncRNAs in the lung tissue of the mice treated with LPS, compared with that of the mice without any treatment (red dots represent up-regulated lncRNAs and blue dots represent down-regulated lncRNAs).The expression of XIST was among the significantly down-regulated lncRNAs. $\mathbf{c}$ The expression of XIST in the lung tissues of the rats in sham group, CLP group and CLP+RAAV-XIST group was detected by RT-qPCR. d The lung W/D weight ratio was

and Bcl-2 and SOD levels were significantly elevated (Fig. 2c-f); conversely, knockdown of XIST exerted the used to indicate the pulmonary edema of the rats in each group. $\mathbf{e}$, f TNF- $\alpha$, IL- $1 \beta$, SOD and MDA levels in the lung tissues of the rats in sham group, CLP group and CLP+RAAV-XIST group were detected, respectively. $\mathbf{g}$ TUNEL assay was carried out to detect the injury of lung tissue of rats. $\mathbf{h}$ HE staining showed that CLP induced lung injury such as alveolar damage, hemorrhage, and thickened alveolar septum, and XIST overexpression ameliorated the CLP-induced lung injury (upper scale $b a r=35 \mu \mathrm{m}$; lower scale $b a r=100 \mu \mathrm{m}$ ). i The subcellular distribution of XIST was examined. All of the experiments were performed in triplicate. $* P<0.05$, $* * P<0.01$, and $* * * P<0.001$

opposite effects (Fig. 2c-f). These data indicated that 

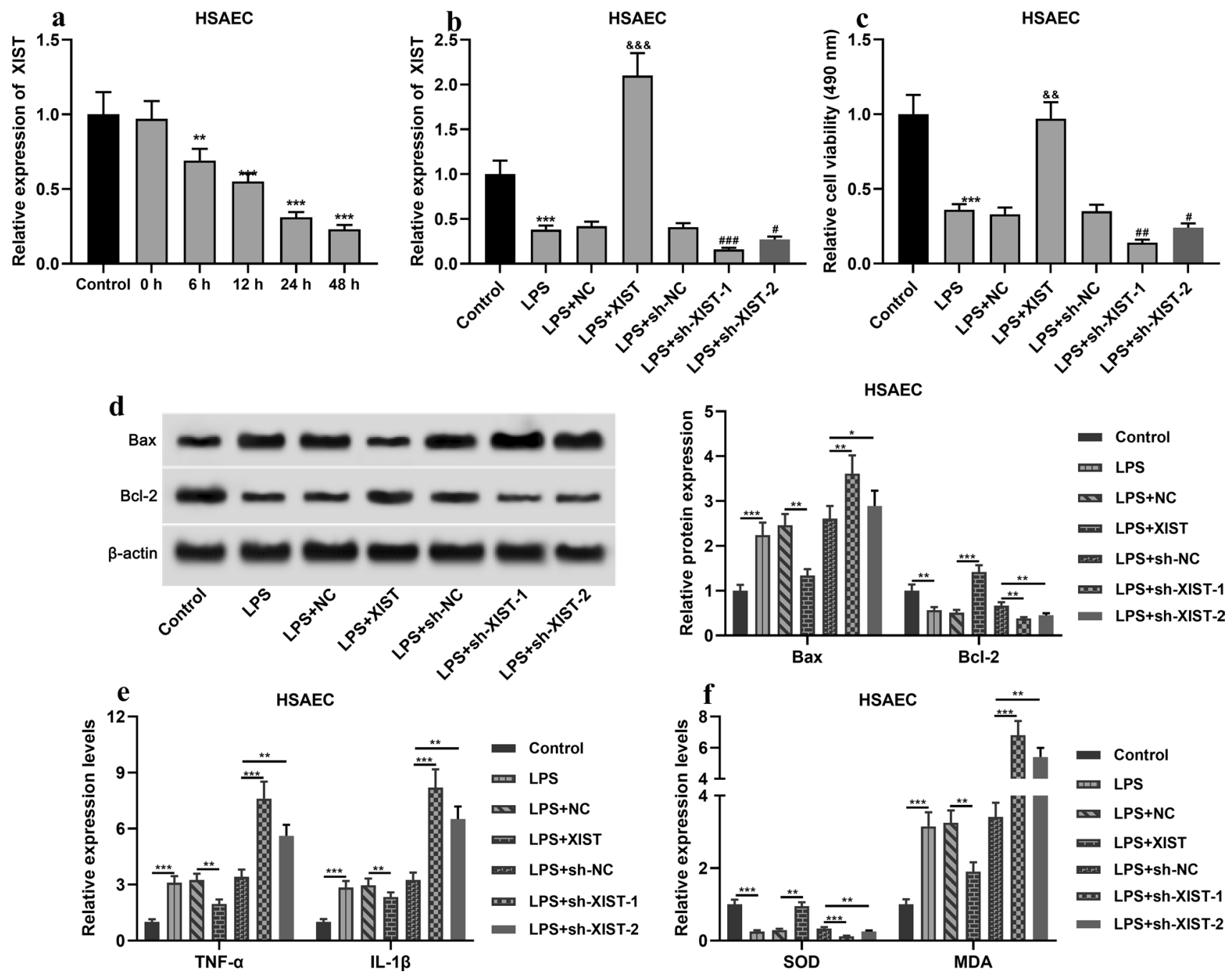

Fig. 2 Effects of XIST on LPS-induced injury of HSAECs. a The expression of XIST in HSAECs treated with LPS was detected by RT-qPCR. b The expression of XIST in HSAECs treated with LPS was detected by RT-qPCR after transfection with XIST overexpression plasmids or XIST shRNAs. c MTT assay was used to detect the viability of HSAECs treated with LPS. d Western blot was applied to detect the expression of apoptosis-related proteins Bax and Bcl-2

XIST counteracted the injury, inflammatory response, and oxidative stress of airway epithelial cells.

\section{XIST directly targets miR-16-5p}

In order to further verify the downstream mechanism of XIST, StarBase (http://starbase.sysu.edu.cn/) and LncBase Predicted V2 (http://carolina.imis.athena-innovation.gr/) were employed for predicting the downstream target miRNAs of XIST, and the results suggested that miR-16-5p was one of the potential targets of XIST (Fig. 3a). By analyzing the miRNAs expression profile in GSE74952, it was found that miR-16-5p was up-regulated in the plasma of in HSAECs treated with LPS after overexpression and knockdown of XIST. e, f The levels of TNF- $\alpha$, IL- $1 \beta$, SOD and MDA in cell culture supernatant were detected, respectively. All of the experiments were performed in triplicate. $* * P<0.01, * * * P<0.001$ vs. control; ${ }^{\& \&} P<0.01,{ }^{\& \& \&} P<0.001$ vs. LPS $+\mathrm{NC},{ }^{\# \#} P<0.01,{ }^{\# \# \#} P<0.001$ vs. LPS + sh-NC

CLP mice, suggesting that sepsis could probably induce the expression of miR-16-5p (Fig. 3b, c). Bioinformatics analysis showed that XIST contained two conserved binding sites of miR-16-5p (Fig. 3d). Dual-luciferase reporter gene assay uncovered that miR-16-5p mimics could reduce the luciferase activity of XIST WT, XIST MUT1 and XIST MUT2 reporters, while it had no significant effect on that of XIST MUT1\&2 reporter (Fig. 3e). In addition, RIP assay and RNA pull-down assay suggested that XIST and miR-16-5p were directly interacted with each other in HSAECs (Fig. 3f, g). These data implied that XIST could function as a molecular sponge to repress miR-16-5p. 

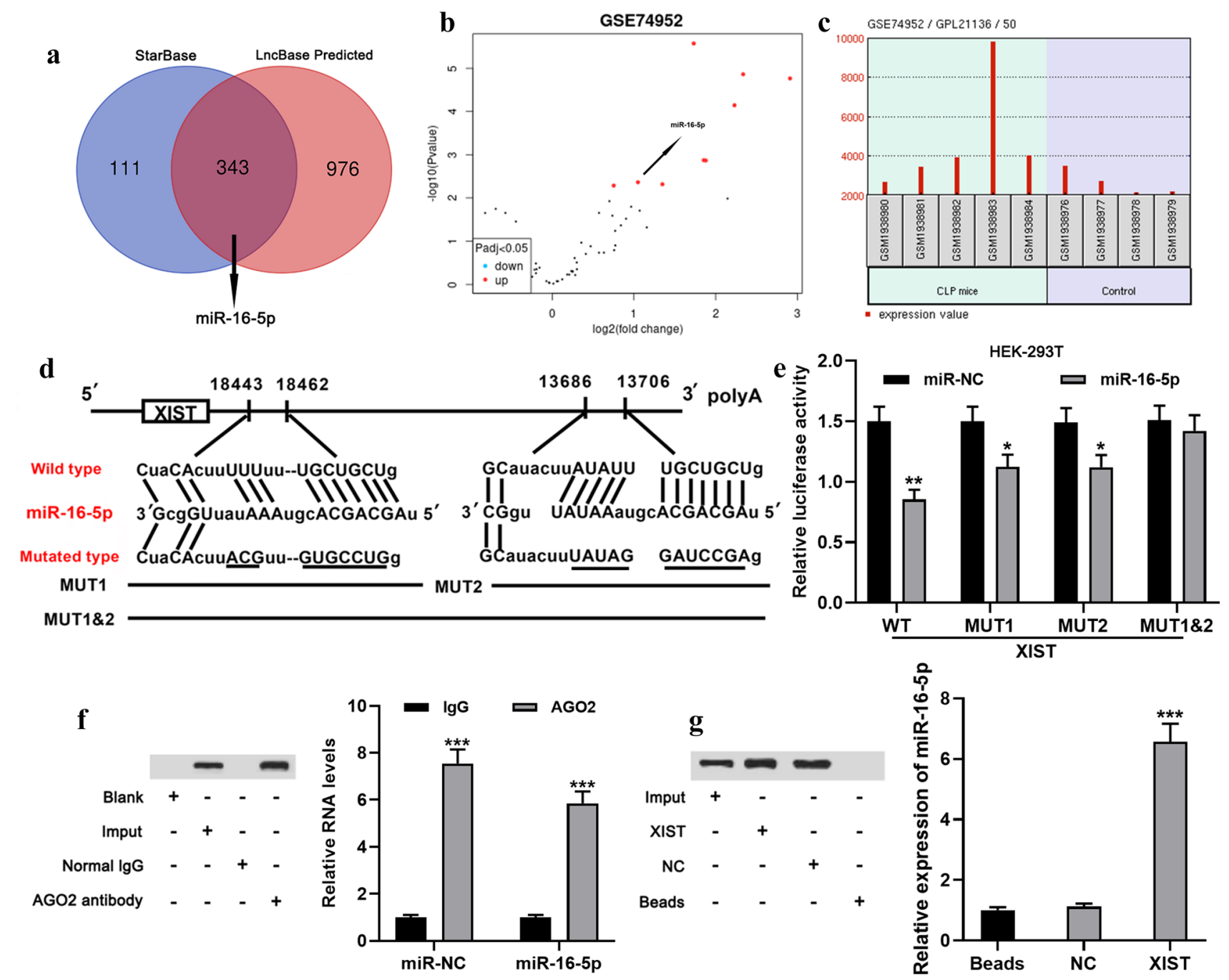

Fig. 3 The interaction between XIST and miR-16-5p. a Venn diagram showed the intersection of the predicted miRNA targets of XIST between StarBase database and LncBase Predicted V2 database. b, c The miRNAs expression dataset GSE74952 was downloaded from GEO database. Red dots indicate the up-regulated miRNAs in the plasma of CLP mice, and the level of miR-16-5p in the plasma of septic mice was elevated. d The biding sites between XIST and

\section{Effects of miR-16-5p on the viability and apoptosis of HSAECs treated with LPS}

To further determine the role of miR-16-5p in septic ALI, we conducted RT-qPCR and demonstrated that the expression of miR-16-5p in HSAECs was significantly up-regulated by LPS treatment in a time-dependent manner (Fig. 4a). Then we successfully transfected miR-16-5p mimics or inhibitors into HSAMCs (Fig. 4b). As shown, miR-16-5p overexpression reduced the viability of HSAMCs, suppressed Bcl-2 and SOD levels, and promoted the apoptosis and increased the levels of Bax, TNF- $\alpha$, IL-1 $\beta$ and MDA (Fig. 4c-f), while miR-16-5p inhibition functioned oppositely (Fig. $4 c-f$ ).
miR-16-5p are displayed. e Dual luciferase report gene assay was performed to verify the targeting relationship between XIST and miR-16-5p. f, $\mathbf{g}$ The binding relationship between XIST and miR$16-5 p$ was detected by RIP assay and RIP pull-down assay, respectively. All of the experiments were performed in triplicate. ${ }^{*} P<0.05$, $* * P<0.01$, and $* * * P<0.001$

These data indicated that miR-16-5p promoted the injury and inflammatory response of airway epithelial cells.

\section{Effects of XIST/miR-16-5p axis on the viability and apoptosis of HSAECs treated with LPS}

Next, miR-16-5p mimics were transfected into HSAECs with XIST overexpression. RT-qPCR showed that the transfection was successful, and XIST overexpression could repress the expression of miR-16-5p mimics (Fig. 5a, b). MTT assay, Western blot and ELISA indicated that after the co-transfection of miR-16-5p mimics, the effects of XIST overexpression on the viability, apoptosis, oxidative 

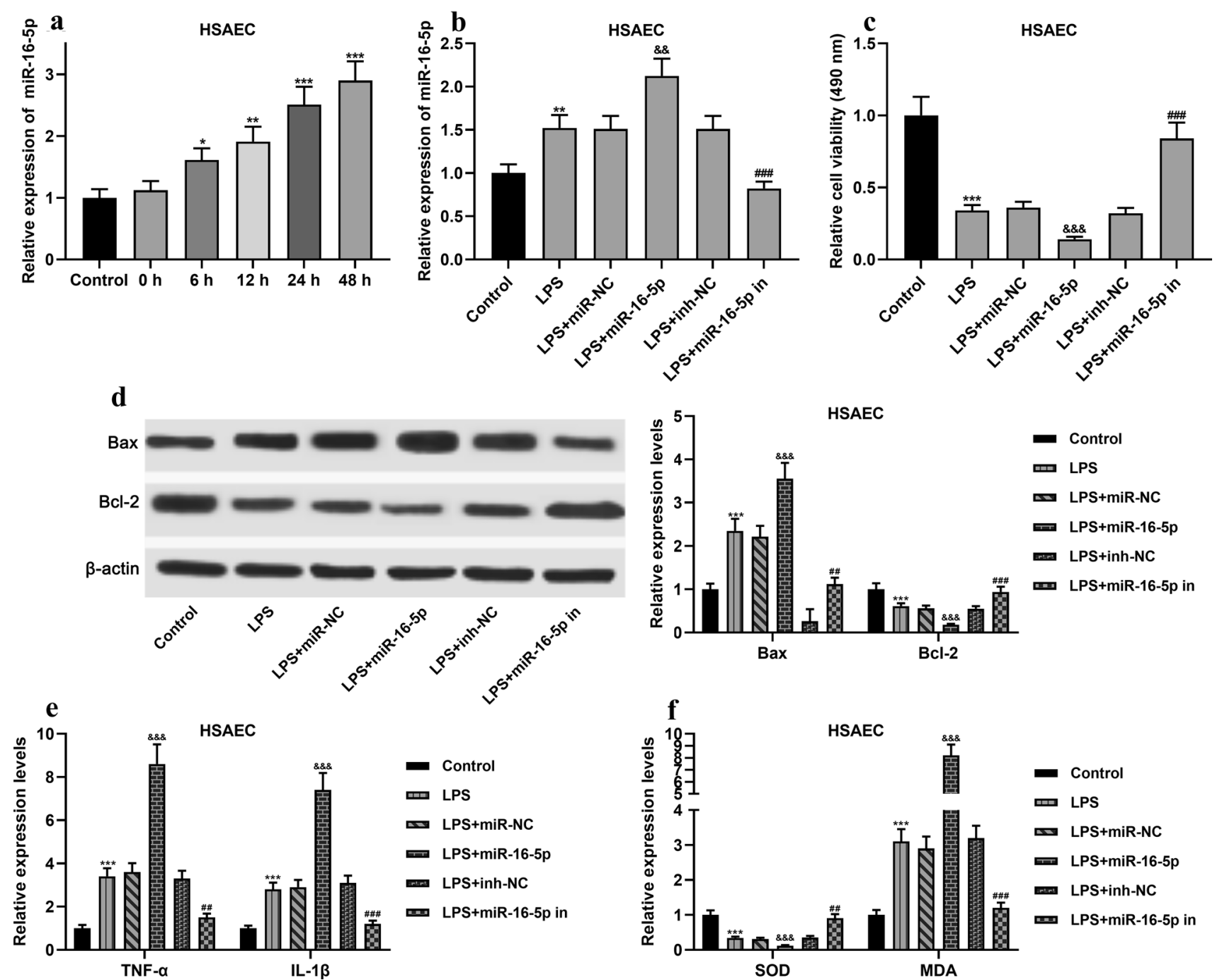

Fig. 4 Effects of overexpression and inhibition of miR-16-5p on LPS-induced injury of HSAECs. a The expression of miR-16-5p in HSAECs treated with LPS was detected by RT-qPCR. b The transfection efficiency of miR-16-5p mimics or inhibitors in HSAECs was validated by RT-qPCR. c MTT assay was used to detect the viability of HSAECs treated with LPS. d Western blot was applied

stress and inflammatory response of HSAECs were reversed (Fig. 5c-f). Collectively, these data implied that XIST could negatively regulate miR-16-5p in HSAECs, and XIST protected airway epithelial cells from sepsis via suppressing miR-16-5p.

\section{Discussion}

Sepsis-induced ALI is regulated by a variety of inflammatory mediators and effector cells. In this process, epithelial cells produce excessive pro-inflammatory cytokines and inflammatory mediators, thereby leading to immune disorders to detect the expression of apoptosis-related proteins Bax and Bcl-2 in HSAECs treated with LPS. e, f The levels of TNF- $\alpha$, IL-1 $\beta$, SOD and MDA in cell culture supernatant were detected. All of the experiments were performed in triplicate. $* P<0.05, * * P<0.01$, ${ }^{* * *} P<0.001$ vs. control; ${ }^{\& \&} P<0.01,{ }^{\& \& \&} P<0.001$ vs. $L P S+\mathrm{miR}-$ NC; ${ }^{\# \#} P<0.01,{ }^{\# \# \#} P<0.001$ vs. LPS + inh-NC

[19]. In addition, previous studies have reported that sepsis is associated not only with inflammatory responses, but also with the excessive production of reactive oxygen species (ROS) and imbalance in the expression of antioxidants $[20,21]$. In the current study, we found that the levels of inflammatory cytokines (TNF- $\alpha$ and IL-1 $\beta$ ) and MDA levels were significantly up-regulated, while SOD levels was significantly down-regulated in septic ALI models; besides, significant pulmonary edema and the reduced viability of HSAECs were observed, which are consistent with the previous reports [19-21].

Reportedly, some lncRNAs are involved in regulating inflammation and oxidative stress. For instance, the 


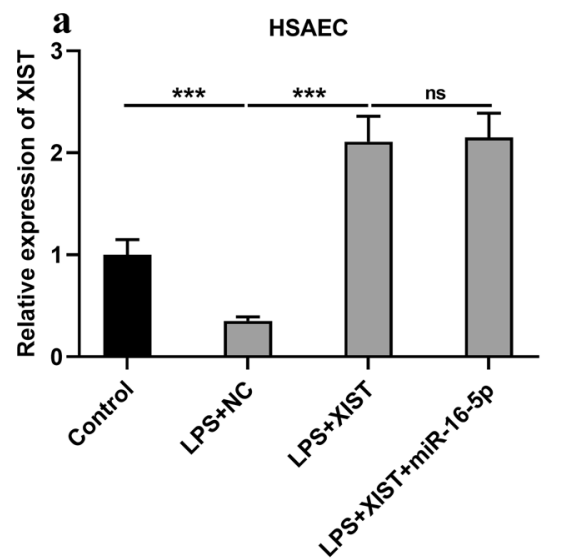

d

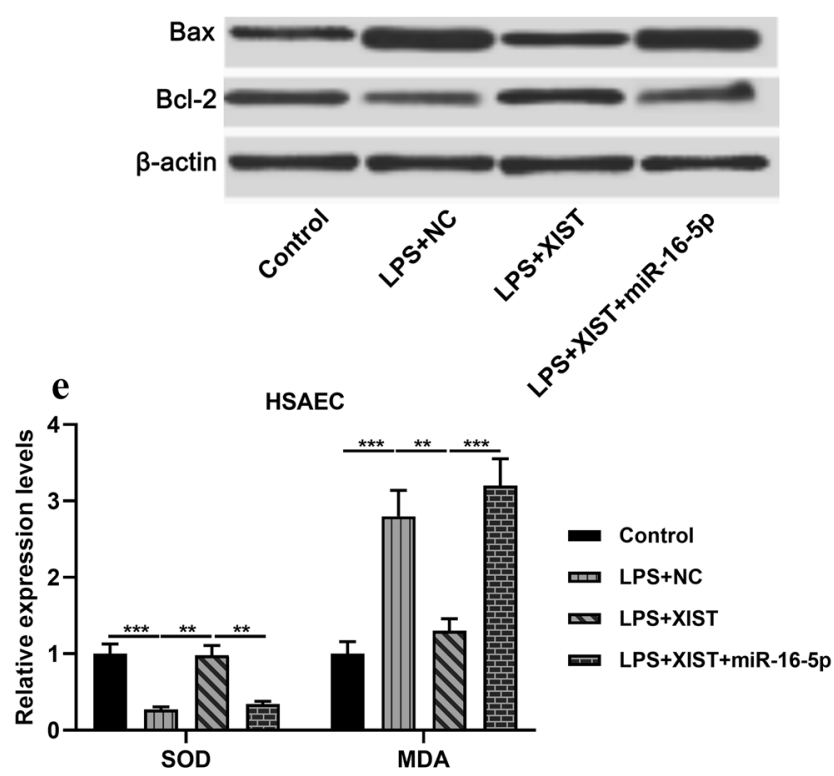

Fig. 5 Effects of XIST/miR-16-5p axis on LPS-induced injury of HSAECs. a, b XIST overexpression and miR-16-5p mimics were co-transfected into HSAECs, and RT-qPCR was applied to detect the expression of XIST and miR-16-5p in HSAECs treated with LPS. c MTT assay was used to detect the viability of HSAECs treated with

increased circulating lncRNA NEAT1 is associated with poor prognosis of patients with sepsis [22]. An another study demonstrates, lncRNA MALAT1 can inhibit ROS levels to prevent LPS-induced dysfunction of cardiac microvascular endothelial cells [23]. Previous studies have confirmed that XIST functions regulate inflammation. For example, in acute pneumonia, XIST is involved in LPS-induced cell injury by regulating JAK/STAT and NF- $\mathrm{\kappa B}$ signal transduction [24]. In addition, XIST regulates bovine mammary epithelial cell inflammatory responses via NF- $\mathrm{BB} / \mathrm{NLRP} 3$ signaling pathway [25]. In this study, we found that the XIST expression was significantly down-regulated in sepsis-induced ALI animal models and cell models. Furthermore, function

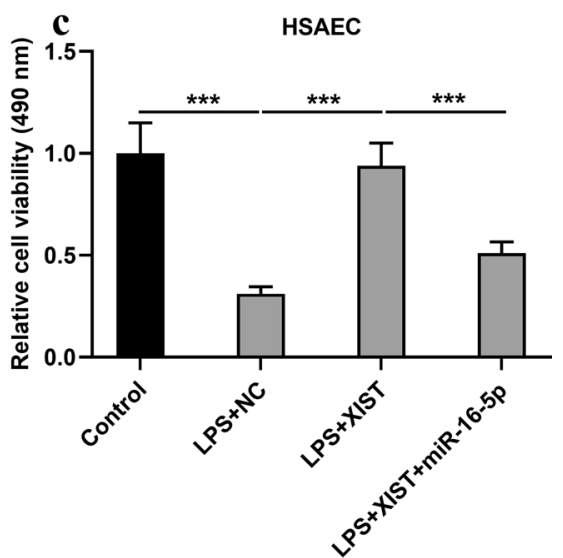

HSAEC
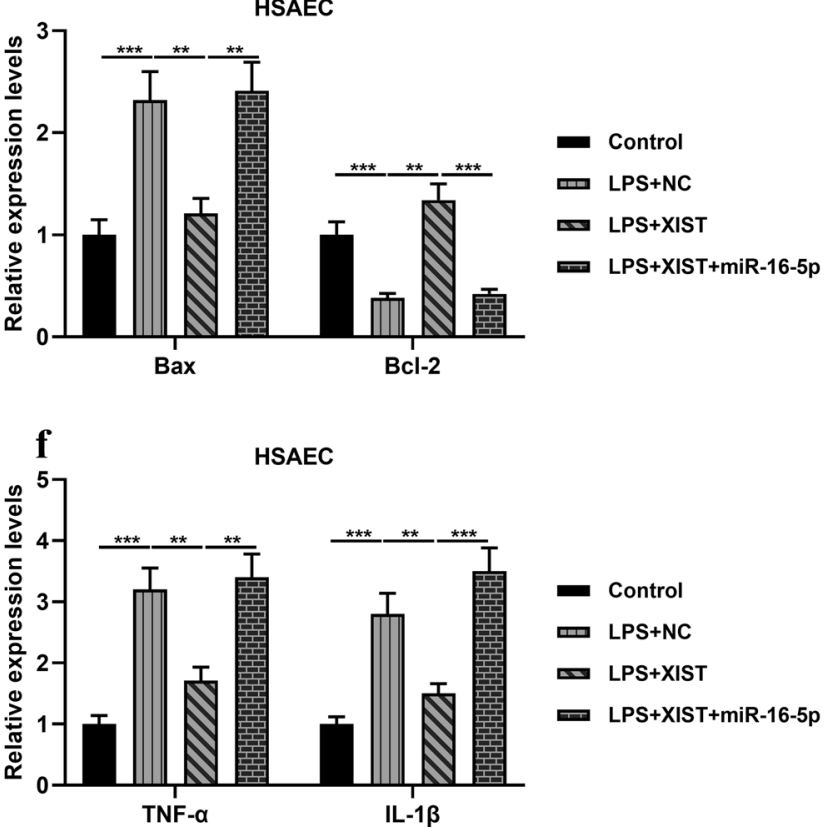

LPS. d Western blot was applied to detect the expression of apoptosis-related proteins Bax and Bcl-2 in HSAECs treated with LPS. e, f The levels of TNF- $\alpha$, IL- $1 \beta$, SOD and MDA in cell culture supernatant were detected, respectively. All of the experiments were performed in triplicate. ${ }^{*} P<0.05$, $* * P<0.01$, and $* * * P<0.001$

experiments confirmed that overexpression of XIST could inhibit lung injury via regulating inflammatory response and oxidative stress. Our data suggest that the down-regulation of XIST contributes to the pathogenesis of sepsis-induced ALI, and restoration of XIST may attenuate the lung injury and improve the prognosis of the patients.

MiRNAs also participate in the development of sepsis. For instance, miRNA-34a can attenuate the sepsis-mediated renal injury by regulating Bcl-2 [26]. Additionally, miRNAs are also involved in sepsis-mediated lung injury. Reportedly, in septic shock, miR-203 can alleviate lung injury by inhibiting VNN1 [27]. In previous studies, miR$16-5 p$ is mainly considered as a tumor suppressor, and it 
can induce cell apoptosis by repressing its target genes such as SMAD3 and Akt3, both of which were crucial modulators in inflammation and lung injury [17, 28-30]. In the present research, we found that the expression of miR-16-5p was significantly up-regulated in lung tissues of animals with sepsis and LPS-induced HSAECs, while inhibition of miR-16-5p could significantly promote the viability and reduce the apoptosis of lung epithelial cells. What is more, inhibition of miR-16-5p could reduce inflammatory response and oxidative stress. These results implied that miR-16-5p could aggravate lung injury during sepsis. Additionally, it is well known that lncRNA can regulate the function of miRNAs by acting as a competitive endogenous RNA. For example, in sepsis-induced lung injury, lncRNA MEG3-4 can indirectly regulate the expression of IL- $1 \beta$ by functioning as a miRNA decoy for miR-138 [31]. The results of the present study showed that XIST could sponge miR-16-5p and inhibit its expression. We also found that overexpression of miR-16-5p could reverse the function of XIST in sepsis-induced ALI. Based on these results, we concluded that XIST could weaken sepsis-mediated ALI by targeting miR-16-5p.

This study has several limitations. First of all, due to the limitation of clinical sample source, itis difficult for us to validate our conclusion in patients with sepsis-induced ALI. In addition, other downstream miRNAs of XIST need to be screened out and validated, which can better clarify how XIST protects lung from injury. What is more, the downstream mechanism of XIST/miR-16-5p axis still remains to be clarified.

To sum up, in this study, we report that XIST is downregulated in sepsis-induced ALI. Overexpression of XIST can significantly alleviate sepsis-mediated lung injury and reduce the apoptosis of lung epithelial cells. We also identify miR-16-5p as an important downstream target of the XIST in sepsis-mediated ALI. Our work helps clarify the mechanism of the pathogenesis of sepsis-induced lung injury and provides promising therapy targets for this deadly disease.

\section{Acknowledgements None}

Authors' contribution Guarantor of integrity of the entire study: YCS; study concepts and design: YCS and LYL; perform the experiments: XFS and YYZ; data analysis: YFS and LYL; manuscript preparation: XFS; manuscript review: LYL and YCS.

Funding This study was financially supported by Henan Provincial Programs for Science and Technology Development (Grant No. 182102310149).

Data availability The data used to support the findings of this study are available from the corresponding author upon a reasonable request.

\section{Declarations}

Conflict of interest The authors declare that there are no conflicts of interest.

Ethical approval The animal study procedures were approved by the Animal Studies Committee of Henan Provincial People's Hospital (Approval No. 2014-A11) and it also followed the ARRIVE guidelines.

\section{References}

1. Dombrovskiy VY, Martin AA, Sunderram J, Paz HL. Rapid increase in hospitalization and mortality rates for severe sepsis in the United States: a trend analysis from 1993 to 2003. Crit Care Med. 2007;35(5):1244-50.

2. Gaieski DF, Edwards JM, Kallan MJ, Carr BG. Benchmarking the incidence and mortality of severe sepsis in the United States. Crit Care Med. 2013;41(5):1167-74.

3. Chen G, Wang Z, Wang D, Qiu C, Liu M, Chen X, Zhang Q, Yan G, Cui Q. LncRNADisease: a database for long-non-coding RNA-associated diseases. Nucleic Acids Res. 2013;41(Database issue):D983-6.

4. Rapicavoli NA, Qu K, Zhang J, Mikhail M, Laberge RM, Chang HY. A mammalian pseudogene lncRNA at the interface of inflammation and anti-inflammatory therapeutics. Elife. 2013;23(2):e00762.

5. Liao K, Xu J, Yang W, You X, Zhong Q, Wang X. The research progress of LncRNA involved in the regulation of inflammatory diseases. Mol Immunol. 2018;101:182-8.

6. Zhang CC, Niu F. LncRNA NEAT1 promotes inflammatory response in sepsis-induced liver injury via the Let-7a/TLR4 axis. Int Immunopharmacol. 2019;22(75):105731.

7. Wang SM, Liu GQ, Xian HB, Si JL, Qi SX, Yu YP. LncRNA NEAT1 alleviates sepsis-induced myocardial injury by regulating the TLR2/NF- $\mathrm{KB}$ signaling pathway. Eur Rev Med Pharmacol Sci. 2019;23(11):4898-907.

8. Wang J, Cai H, Dai Z, Wang G. Down-regulation of lncRNA XIST inhibits cell proliferation via regulating miR-744/ RING1 axis in non-small cell lung cancer. Clin Sci (Lond). 2019;133(14):1567-79.

9. Shen J, Hong L, Yu D, Cao T, Zhou Z, He S. LncRNA XIST promotes pancreatic cancer migration, invasion and EMT by sponging miR-429 to modulate ZEB1 expression. Int J Biochem Cell Biol. 2019;113:17-26.

10. Li J, Wei L, Han Z, Chen Z, Zhang Q. Long non-coding RNA $\mathrm{X}$-inactive specific transcript silencing ameliorates primary graft dysfunction following lung transplantation through microRNA21-dependent mechanism. EBioMedicine. 2020;52:102600.

11. Bartel DP. MicroRNAs: genomics, biogenesis, mechanism, and function. Cell. 2004;116(2):281-97.

12. Alvarez-Garcia I, Miska EA. MicroRNA functions in animal development and human disease. Development. 2005;132(21):4653-62.

13. Sonkoly E, Pivarcsi A. Advances in microRNAs: implications for immunity and inflammatory diseases. J Cell Mol Med. 2009; 13(1):24-38.

14. Kingsley SMK, Bhat BV. Role of microRNAs in sepsis. Inflamm Res. 2017;66(7):553-69.

15. Zhang W, Jia J, Liu Z, Si D, Ma L, Zhang G. Circulating microRNAs as biomarkers for sepsis secondary to pneumonia diagnosed via sepsis 3.0. BMC Pulm Med. 2019;19(1):93.

16. Zhang J, Song Y, Zhang C, Zhi X, Hualin Fu, Ma Y, et al. Circulating miR-16-5p and miR-19b-3p as two novel potential 
biomarkers to indicate progression of gastric cancer. Theranostics. 2015;5(7):733-45.

17. Zhang H, Yang K, Ren T, Huang Y, Tang X, Guo W. miR-16-5p inhibits chordoma cell proliferation, invasion and metastasis by targeting Smad3. Cell Death Dis. 2018;9(6):680.

18. Yang X, Lu GP, Cai XD, Lu ZJ, Kissoon N. Alterations of complex IV in the tissues of a septic mouse model. Mitochondrion. 2019;49:89-96.

19. Sapru A, Flori H, Quasney MW, Dahmer MK, et al. Pathobiology of acute respiratory distress syndrome. Pediatr Crit Care Med. 2015;16(5 Suppl 1):S6-22.

20. Wang X, An X, Wang X, Hu X, Bi J, Tong L, Yang D, Song Y, Bai C. Peroxiredoxin 6 knockout aggravates cecal ligation and puncture-induced acute lung injury. Int Immunopharmacol. 2019;68:252-8.

21. Bai YY, Yan D, Zhou HY, Li WX, Lou YY, Zhou XR, Qian LB, Xiao C. Betulinic acid attenuates lipopolysaccharide-induced vascular hyporeactivity in the rat aorta by modulating Nrf2 antioxidative function. Inflammopharmacology. 2020;28(1):165-74.

22. Huang Q, Huang C, Luo Y, He F, Zhang R. Circulating lncRNA NEAT1 correlates with increased risk, elevated severity and unfavorable prognosis in sepsis patients. Am J Emerg Med. 2018;36(9):1659-63.

23. Yu Z, Rayile A, Zhang X, Li Y, Zhao Q. Ulinastatin protects against lipopolysaccharide-induced cardiac microvascular endothelial cell dysfunction via downregulation of lncRNA MALAT1 and EZH2 in sepsis. Int J Mol Med. 2017;39(5):1269-76.

24. Zhang Y, Zhu Y, Gao G, Zhou Z. Knockdown XIST alleviates LPS-induced WI-38 cell apoptosis and inflammation injury via targeting miR-370-3p/TLR4 in acute pneumonia. Cell Biochem Funct. 2019;37(5):348-58.

25. Ma M, Pei Y, Wang X, Feng J, Zhang Y, Gao MQ. LncRNA XIST mediates bovine mammary epithelial cell inflammatory response via NF- $\mathrm{kB} / \mathrm{NLRP} 3$ inflammasome pathway. Cell Prolif. 2019;52(1):e12525.

26. Jiang ZJ, Zhang MY, Fan ZW, Sun WL, Tang Y. Influence of lncRNA HOTAIR on acute kidney injury in sepsis rats through regulating miR-34a/Bcl-2 pathway. Eur Rev Med Pharmacol Sci. 2019;23(8):3512-9.

27. Ling L, Lu HT, Wang HF, Shen MJ, Zhang HB. MicroRNA-203 acts as a potent suppressor in septic shock by alleviating lung injury via inhibition of VNN1. Kidney Blood Press Res. 2019;44:1-18.

28. Ruan L, Qian X. MiR-16-5p inhibits breast cancer by reducing AKT3 to restrain NF- $\mathrm{BB}$ pathway. Biosci Rep. 2019;39(8):BSR20191611.

29. Zhao J, Shi W, Wang YL, Chen H, Bringas P Jr, Datto MB, Frederick JP, Wang XF, Warburton D. Smad3 deficiency attenuates bleomycin-induced pulmonary fibrosis in mice. Am J Physiol Lung Cell Mol Physiol. 2002;282(3):L585-93.

30. Yang SJ, Chen HM, Hsieh CH, Hsu JT, Yeh CN, Yeh TS, Hwang TL, Jan YY, Chen MF. Akt pathway is required for oestrogenmediated attenuation of lung injury in a rodent model of ceruleininduced acute pancreatitis. Injury. 2011;42(7):638-42.

31. Li R, Fang L, Pu Q, Bu H, Zhu P, Chen Z, Yu M, Li X, Weiland T, Bansal A, Ye SQ, Wei Y, Jiang J, Wu M. MEG3-4 is a miRNA decoy that regulates IL-1 $\beta$ abundance to initiate and then limit inflammation to prevent sepsis during lung infection. Sci Signal. 2018;11(536):eaao2387.

Publisher's Note Springer Nature remains neutral with regard to jurisdictional claims in published maps and institutional affiliations. 